\title{
Eribulin Suppressed Cisplatinum- and Doxorubicin-resistant Recurrent Lung Metastatic Osteosarcoma in a Patient-derived Orthotopic Xenograft Mouse Model
}

\author{
TASUKU KIYUNA ${ }^{1,2,3}$, YASUNORI TOME ${ }^{3}$, KENTARO MIYAKE $^{1,2}$, \\ TAKASHI MURAKAMI ${ }^{1,2}$, HIROMICHI OSHIRO ${ }^{1,2,3}$, KENTARO IGARASHI $^{1,2}$, \\ KEI KAWAGUCHI ${ }^{1,2}$, JOHN HSU ${ }^{1}$, MANISH SINGH ${ }^{4}$, YUNFENG LI ${ }^{5}$, SCOTT NELSON ${ }^{5}$, \\ MICHAEL BOUVET ${ }^{2}$, SHREE RAM SINGH ${ }^{6}$, FUMINORI KANAYA ${ }^{3}$ and ROBERT M. HOFFMAN ${ }^{1,2}$ \\ ${ }^{1}$ AntiCancer Inc., San Diego, CA, U.S.A.; \\ ${ }^{2}$ Department of Surgery, University of California, San Diego, CA, U.S.A.; \\ ${ }^{3}$ Department of Orthopedic Surgery, Graduate School of Medicine, University of the Ryukyus, Okinawa, Japan; \\ ${ }^{4}$ Mouse Cancer Genetics Program, National Cancer Institute, Frederick, MD, U.S.A.; \\ ${ }^{5}$ Department of Pathology, University of California, Los Angeles, CA, U.S.A.; \\ ${ }^{6}$ Basic Research Laboratory, National Cancer Institute, Frederick, MD, U.S.A.
}

\begin{abstract}
Background: Osteosarcoma is a recalcitrant disease treated with surgery and intensive chemotherapy as standard. The 5-year survival rate of patients with relapsed and lung metastatic osteosarcoma is as low as 20\%. Materials and Methods: A 16-year-old patient developed left distal femoral high-grade osteosarcoma and underwent cisplatinumbased neoadjuvant chemotherapy and surgery. From the resected tumor, a patient-derived orthotopic xenograft (PDOX) model was established in the femur of nude mice. PDOX models were randomized into the following groups: untreated control, or treatment with doxorubicin $(3 \mathrm{mg} / \mathrm{kg}$, i.p., weekly for 14 days), sunitinib (40 mg/kg, oral gavage, daily for 14 days), pazopanib (100 mg/ $/ \mathrm{kg}$, oral gavage, daily for 14 days), temozolomide $(25 \mathrm{mg} / \mathrm{kg}$, oral gavage, daily for 14 days) , and eribulin (1.5 mg/kg, i.p., daily for 14 days). Tumor volume and body weight were monitored twice a week. Results: The osteosarcoma PDOX was resistant to doxorubicin, sunitinib, and pazopanib. In contrast, eribulin
\end{abstract}

Correspondence to: Robert M. Hoffman, Ph.D., AntiCancer, Inc., 7917 Ostrow Street, San Diego, CA 92111, U.S.A. Tel: +1 8586542555, Fax: +1 8582684175, e-mail: all@anticancer.com; Yasunori Tome, MD, Ph.D., Department of Orthopedic Surgery, Graduate School of Medicine, University of the Ryukyus, Okinawa, Japan. E-mail: yash_toume@hotmail.com; Shree Ram Singh, Ph.D., Basic Research Laboratory, National Cancer Institute, Frederick, MD 21702, U.S.A. Tel: +1 3018467331, e-mail: singhshr@mail.nih.gov

Key Words: Osteosarcoma, patient-derived orthotopic xenograft, PDOX, metastasis, eribulin, temozolomide, sunitinib, pazopanib, doxorubicin, precision medicine. and temozolomide arrested tumor growth. Conclusion: This study demonstrated the utility of the PDOX model in allowing effective from non-effective drugs to be distinguished in a model in which the tumor was growing on the organ corresponding to that of the patient.

Osteosarcoma is the most common and still recalcitrant sarcoma arising from bone, and affects mostly children and adolescents (1-3). First-line treatment for osteosarcoma includes surgery and intensive chemotherapy such as MAP (methotrexate, doxorubicin, cisplatinum), but the 5-year survival rate of patients with relapsed and lung metastatic case is under $20 \%$ (1). Therefore, improved precision therapy is needed for this disease. To meet this goal, we developed patient-derived orthotopic xenograft (PDOX) mouse model which can be used for all cancer types (4).

Eribulin, a nontaxane microtubule targeting agent, destabilizes microtubules by targeting microtubule plus ends and inhibiting microtubule elongation $(5,6)$. Eribulin has shown activity against breast cancer and advanced soft-tissue sarcoma (7-9). Recently, it was shown that low-dose eribulin administration can reduce lung metastasis with few sideeffects (10).

We previously demonstrated the efficacy of eribulin on an Ewing's sarcoma PDOX model (11), but to our knowledge there are no studies that have evaluated eribulin in a patientlike model of osteosarcoma.

In our previous studies, we demonstrated the efficacy of temozolomide on several sarcoma PDOX models (12-15). In a previous study, a PDOX model of a lung-metastatic osteosarcoma was established $(13,16)$. We found that this 

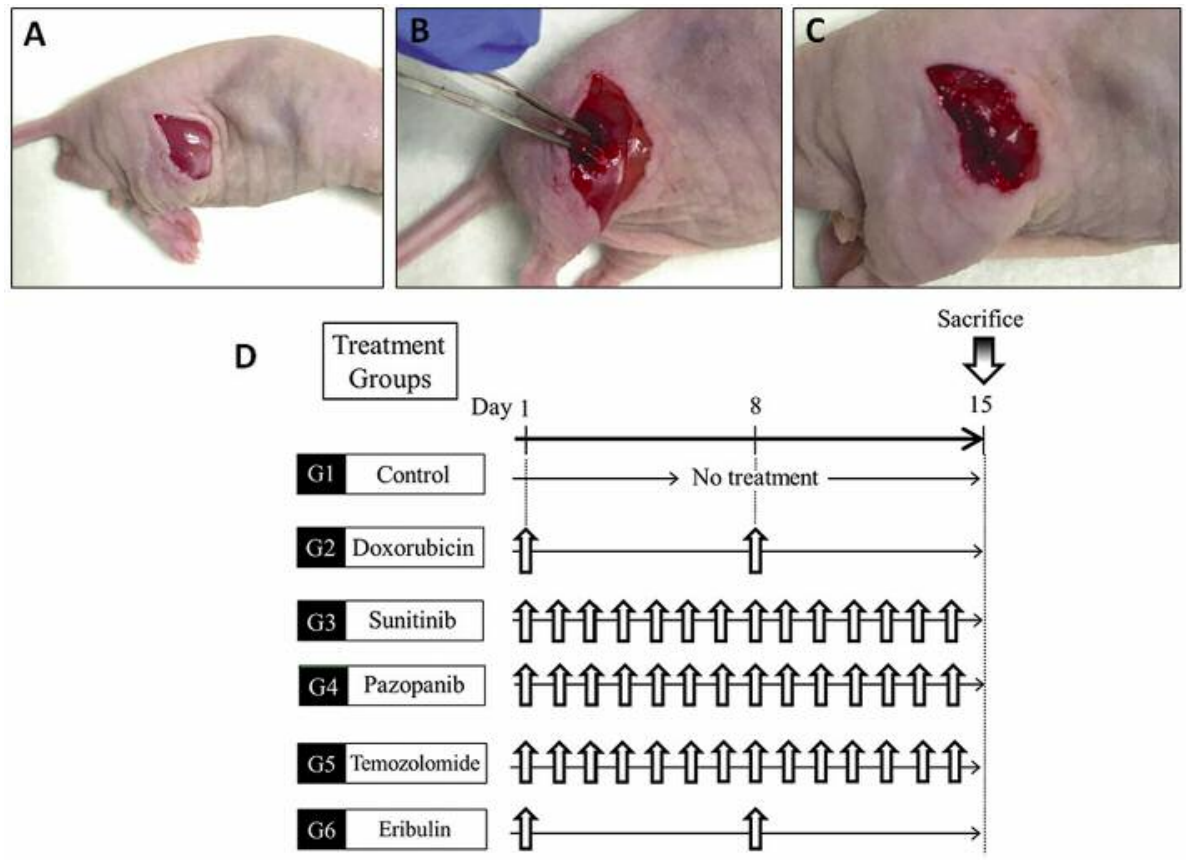

Figure 1. Establishing osteosarcoma patient-derived orthotopic xenograft (PDOX) model and treatment protocols. A: Establishment of osteosarcoma PDOX nude-mouse model. B: Treatment protocol of osteosarcoma PDOX nude-mouse model. Each group comprised seven mice.

PDOX model was sensitive to temozolomide alone (13) and the combination of temozolomide and irinotecan (17), as well as tumor-targeting Salmonella typhimurium A1-R (16, 18).

In the present study, we evaluated efficacy of eribulin in the lung-metastatic osteosarcoma PDOX model resistant to standard therapy.

\section{Materials and Methods}

Mice. Athymic nu/nu nude mice (AntiCancer Inc., San Diego, CA, USA), 4-6 weeks old, were used $(13,16)$. All animal studies were performed with an AntiCancer Inc. Institutional Animal Care and Use Committee (IACUC)-protocol specifically approved for this study and in accordance with the principals and procedures outlined in the National Institute of Health (NIH) Guide for the Care and Use of Animals under Assurance Number A3873-1 $(13,16)$. Detailed protocols for animal handing, anesthesia, surgery, and animal husbandry are described elsewhere $(13,16)$.

Patient-derived tumor. A 16-year-old patient with localized left distal femoral high-grade osteosarcoma underwent neoadjuvant and adjuvant MAP chemotherapy. One year later, the osteosarcoma recurred with three bilateral metachronous pulmonary metastases. Therefore, the patient was treated with curative surgery at the Division of Surgical Oncology, University of California, Los Angeles. Written informed consent was obtained from the patient as part of a University of California, Los Angeles Institutional Review Board (IRB\#10-001857) approved protocol to establish a PDOX model $(13,16)$.
Establishment of the PDOX osteosarcoma model. In previous work, a lung metastasis from the osteosarcoma patient was established subcutaneously in mice $(13,16)$. Procedures to harvest tumors, and preparing fragments were previously described $(13,16)$. After nude mice $(n=42)$ were anesthetized, a $10 \mathrm{~mm}$ skin incision was made on the right thigh, the vastus lateralis muscle was opened, and the biceps femoris muscle was split to reach the distal femur. An incision was made in the lateral patello-femoral ligament, sparing the knee joint and then the lateral condyle of the femur was resected (13). A single 3 to $4 \mathrm{~mm}$ tumor fragment was implanted orthotopically into the space to establish a PDOX model (13). The muscle and wound were closed with 6-0 nylon suture (13) (Figure 1A).

Treatment study design in the PDOX osteosarcoma model. Doses for treatment were determined based on our previous publications (12-17). Two weeks after orthotopic implantation, mice with tumors that had reached $70 \mathrm{~mm}^{3}$ volume were allocated to each group and treatment was started (Figure 1B).

PDOX mouse models were randomized into the following groups of seven mice each: Group 1, untreated control; group 2, treated with doxorubicin ( $3 \mathrm{mg} / \mathrm{kg}$, i.p., weekly); group 3, treated with sunitinib $(40 \mathrm{mg} / \mathrm{kg}$, oral gavage, daily); group 4 , treated with pazopanib (100 mg/kg, oral gavage, daily); group 5, treated with temozolomide $(25 \mathrm{mg} / \mathrm{kg}$, oral gavage, daily); group 6 , treated with eribulin $(1.5 \mathrm{mg} / \mathrm{kg}$, i.p., daily). The detailed protocol to measure tumor length, width, and mouse body weight were measured twice a week as previously described $(13,16)$. Tumor volume was calculated based on our previous publications $(13,16)$. Data are presented as mean \pm standard deviation $(\mathrm{SD})$. All mice included in this study were sacrificed on day 15 , and tumors were harvested for further histological evaluation. 

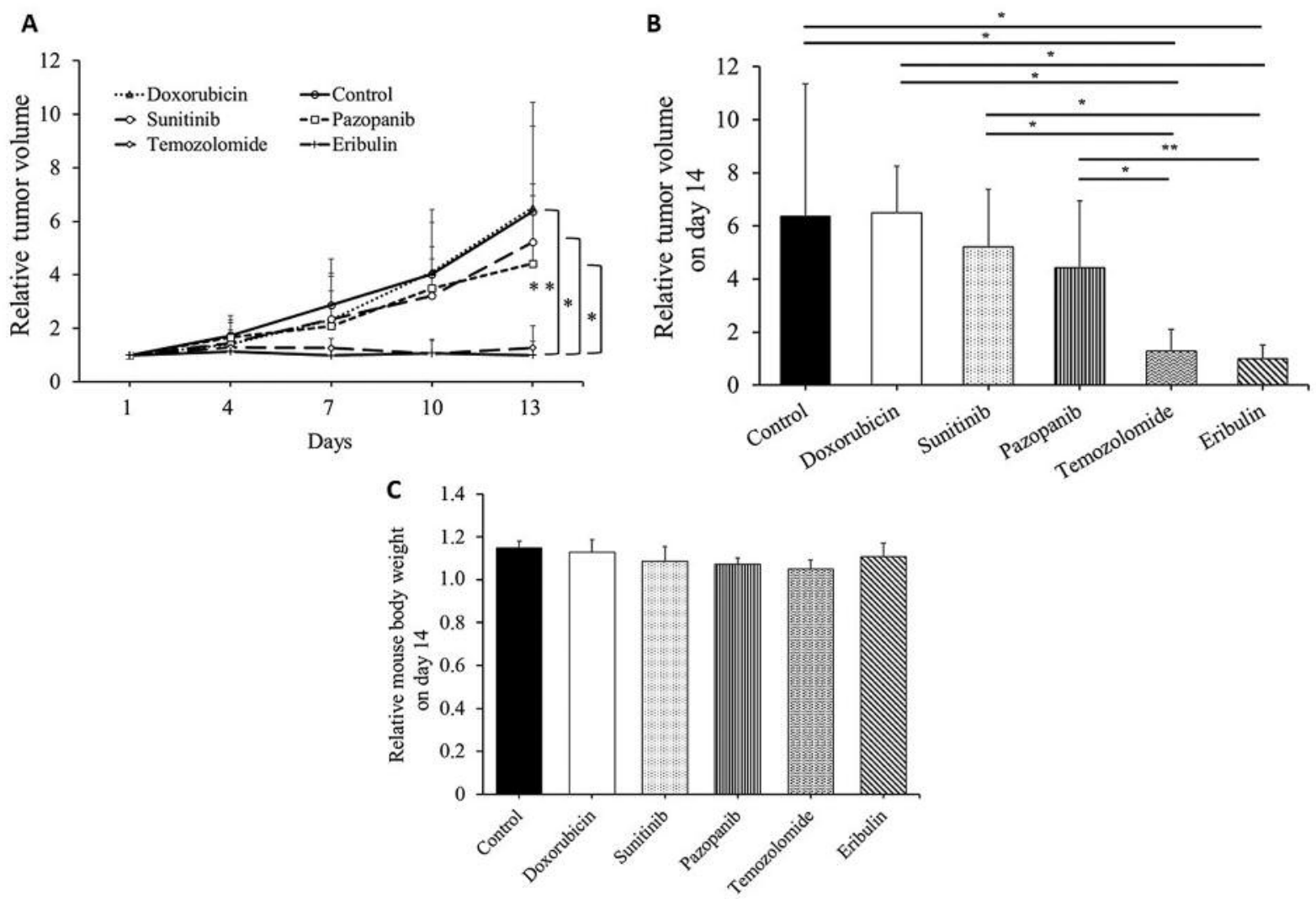

Figure 2. Tumor volume and mouse weight. A: Longitudinal osteosarcoma patient-derived orthotopic xenograft (PDOX) tumor growth. Line graphs show tumor volume at the indicated times relative to that at the start of treatment. B: Tumor volume on day 14 relative to that at the start of treatment of the osteosarcoma for each group. C: Bar graphs showing relative body weight of mice treated with each agent compared to that of the control at day 14 to day 0 of treatment. Significantly different at $* p<0.05$ and $* * p<0.01$.
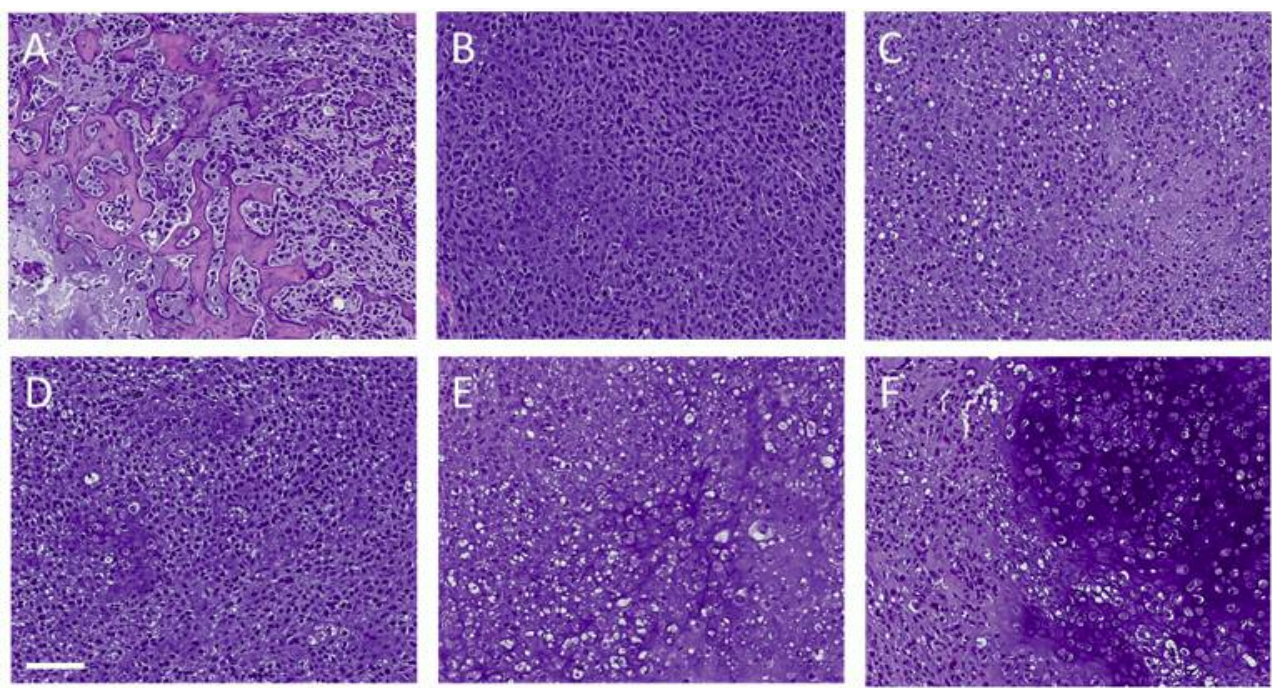

Figure 3. Tumor histology of untreated control group (A), and groups treated with doxorubicin $(B)$, sunitinib $(C)$, pazopanib $(D)$ temozolomide $(E)$ and eribulin $(F)$. Histological sections were stained with hematoxylin and eosin. White bar $=100 \mu \mathrm{m}$. 
Tumor histological examination. Fresh tumor samples from each treatment group including control were fixed for histological analysis. The detailed protocols for fresh tumor samples fixation, sectioning and staining were previously described $(13,16)$. Histological examination and image acquisition were previously described $(13,16)$.

Statistical analysis. Statistical analyses were performed with JMP pro version 12 (SAS Institute, Inc., Cary, NC, USA) for all statistical analyses. The relative tumor volumes and relative body weights of mice are expressed as mean \pm SD. Significant differences for continuous variables were determined using the Steel-Dwass test for multiple comparison. A probability value of less than 0.05 was considered statistically significant.

\section{Results and Discussion}

Figure 2A shows longitudinal relative tumor volume, and Figure 2B compares relative tumor growth on day 14 of treatment. The tumors in the control group had grown approximately 6-fold by day 14. The doxorubicin-treated group did not show significant tumor growth inhibition on day 14. Moreover, neither sunitinib nor pazopanib showed significant efficacy compared to the control group on day 14. In contrast, temozolomide significantly inhibited tumor growth compared to the untreated control $(\sim 80 \%)$, and groups treated with doxorubicin, sunitinib, or pazopanib on day $14(p<0.01, p<0.01, p<0.05$ and $p<0.05$, respectively); eribulin resulted in near tumor regression on day 14 including one complete response. There was no significant body weight loss in any group (Figure 2C).

Figure 3 shows representative tumor histology of all groups. The untreated control group showed anaplastic hypercellular populations, displaying coarse and hyperchromatic nuclei with neoplastic osteoid formation (Figure 3A). Doxorubicin-, and pazopanib-treated PDOX tumors (Figure 3B and D, respectively), comprising viable tumor cells without obvious necrotic areas, were similar to the untreated control group, while sunitinib-treated PDOX tumors showed necrotic areas (Figure 3C). Temozolomide-, and eribulin-treated PDOX tumors had extensive necrotic areas (Figure 3E and F, respectively).

The PDOX model of the cisplatin-resistant lung-metastatic osteosarcoma was previously been shown to be cisplatinresistant as was the original tumor of the patient $(13,16)$, demonstrating the precision of the PDOX model. We previously showed that temozolomide is effective in the PDOX model, both alone and in combination with irinotecan (17). Recent studies suggest that eribulin can be combined with a broad set of anticancer agents (19). The antitumor activity of eribulin is achieved via modulating the tumor microenvironment and vascular remodeling (20). The present report demonstrates that eribulin is possibly more effective than temozolomide on osteosarcoma PDOX and also more effective than the so-called molecular targeting drugs sunitinib and pazopanib, or first-line therapy doxorubicin.

\section{Ethical Approval}

The study was carried out under an AntiCancer Inc. Institutional Animal Care and Use Committee-approved protocol.

\section{Authors' Contributions}

TK conceived and carried out the experiments with the collaboration of YT, KM, TM, HO, KI, KK, and JH. TK, YT, KM, TM, HO, KI, KK, JH, SN, MB, SRS, FK and RMH were involved in data analysis and interpretation. RMH, SRS, MS and TK reviewed and revised the article.

\section{Conflicts of Interest}

TK, YT, KM, TM, HO, KI, KK and RMH are or were unsalaried associates of AntiCancer Inc. AntiCancer Inc. uses orthotopic mouse models for contract research. The Authors have no conflicts of interest relevant to this study to disclose.

\section{References}

1 Shaikh AB, Li F, Li M, He B, He X, Chen G, Guo B, Li D, Jiang F, Dang L, Zheng S, Liang C, Liu J, Lu C, Liu B, Lu J, Wang $\mathrm{L}, \mathrm{Lu} \mathrm{A}$ and Zhang G: Present advances and future perspectives of molecular targeted therapy for osteosarcoma. Int J Mol Sci 17: 506, 2016. PMID: 27058531. DOI: 10.3390/ijms17040506

2 Barøy T, Chilamakuri CS, Lorenz S, Sun J, Bruland ØS, Myklebost $\mathrm{O}$ and Meza-Zepeda LA: Genome analysis of osteosarcoma progression samples identifies $F G F R 1$ overexpression as a potential treatment target and CHM as a candidate tumor suppressor gene. PLoS One 11: e0163859, 2016. PMID: 27685995. DOI: 10.1371/journal.pone.0163859

3 Berner K, Hall KS, Monge OR, Weedon-Fekjær H, Zaikova O and Bruland ØS: Prognostic factors and treatment results of high-grade osteosarcoma in Norway: A scope beyond the "classical" patient. Sarcoma 2015: 516843, 2015. PMID: 25784831. DOI: $10.1155 / 2015 / 516843$

4 Hoffman RM: Patient-derived orthotopic xenografts: Better mimic of metastasis than subcutaneous xenografts. Nat Rev Cancer 15: 451-452, 2015. PMID: 26422835. DOI: 10.1038/nrc3 972

5 Dumontet $\mathrm{C}$ and Jordan MA: Microtubule-binding agents: A dynamic field of cancer therapeutics. Nat Rev Drug Discov 9: 790-803, 2010. PMID: 20885410. DOI: 10.1038/nrd3253

6 Smith JA, Wilson L, Azarenko O, Zhu X, Lewis BM, Littlefield BA and Jordan MA: Eribulin binds at microtubule ends to a single site on tubulin to suppress dynamic instability. Biochemistry 49: 1331-1337, 2010. PMID: 20030375. DOI: 10.1021/bi901810u

7 Kaufman PA, Awada A, Twelves C, Yelle L, Perez EA, Velikova G, Olivo MS, He Y, Dutcus CE and Cortes J: Phase III Openlabel randomized study of eribulin mesylate versus capecitabine in patients with locally advanced or metastatic breast cancer previously treated with an anthracycline and a taxane. J Clin Oncol 33: 594-601, 2015. PMID: 25605862. DOI: 10.1200/ JCO.2013.52.4892

8 Schöffski P, Chawla S, Maki RG, Italiano A, Gelderblom H, Choy E, Grignani G, Camargo V, Bauer S, Rha SY, Blay JY, Hohenberger P, D'Adamo D, Guo M, Chmielowski B, Le Cesne 
A, Demetri GD and Patel SR: Eribulin versus dacarbazine in previously treated patients with advanced liposarcoma or leiomyosarcoma: a randomised, open-label, multicentre, phase 3 trial. Lancet 387: 1629-1637, 2016. PMID: 26874885. DOI: 10.1016/S0140-6736(15)01283-0

9 Kawai A, Araki N, Naito Y, Ozaki T, Sugiura H, Yazawa Y, Morioka H, Matsumine A, Saito K, Asami S and Isu K: Phase 2 study of eribulin in patients with previously treated advanced or metastatic soft-tissue sarcoma. Jpn J Clin Oncol 47(2): 137-144, 2017. PMID: 28173193. DOI: 10.1093/jjco/hyw 175

10 Watanabe K, Yui Y, Sasagawa S, Suzuki K, Kanamori M, Yasuda $\mathrm{T}$ and Kimura T: Low-dose eribulin reduces lung metastasis of osteosarcoma in vitro and in vivo. Oncotarget 10(2): 161-174, 2019. PMID: 30719211. DOI: 10.18632/oncotarget.26536

11 Miyake K, Murakami T, Kiyuna T, Igarashi K, Kawaguchi K, Li Y, Singh AS, Dry SM, Eckardt MA, Hiroshima Y, Momiyama M, Matsuyama R, Chishima T, Endo I, Eilber FC and Hoffman RM: Eribulin regresses a doxorubicin-resistant Ewing's sarcoma with a $F U S-E R G$ fusion and $C D K N 2 A$-deletion in a patientderived orthotopic xenograft (PDOX) nude mouse model. J Cell Biochem 119(1): 967-972, 2018. PMID: 28681998. DOI: $10.1002 /$ jcb. 26263

12 Kiyuna T, Murakami T, Tome Y, Igarashi K, Kawaguchi K, Miyake K, Miyake M, Li Y, Nelson SD, Dry SM, Singh AS, Russell TA, Singh SR, Kanaya F, Eilber FC and Hoffman RM: Doxorubicin-resistant pleomorphic liposarcoma with PDGFRA gene amplification is targeted and regressed by pazopanib in a patient-derived orthotopic xenograft mouse model. Tissue Cell 53: 30-36, 2018. PMID: 30060824. DOI: 10.1016/j.tice.2018. 05.010

13 Igarashi K, Murakami T, Kawaguchi K, Kiyuna T, Miyake K, Zhang Y, Nelson SD, Dry SM, Li Y, Yanagawa J, Russell TA, Singh AS, Tsuchiya H, Elliott I, Eilber FC and Hoffman RM: A patient-derived orthotopic xenograft (PDOX) mouse model of a cisplatinum-resistant osteosarcoma lung metastasis that was sensitive to temozolomide and trabectedin: implications for precision oncology. Oncotarget 8: 62111-62119, 2017. PMID: 28977930. DOI: $10.18632 /$ oncotarget.19095

14 Kiyuna T, Tome Y, Murakami T, Miyake K, Igarashi K, Kawaguchi $\mathrm{K}$, Oshiro H, Higuchi T, Miyake M, Sugisawa N, Zhang Z, Razmjooei S, Wangsiricharoen S, Chmielowski B, Nelson SD, Russell TA, Dry SM, Li Y, Eckardt MA, Singh AS, Chawla S, Kanaya F, Eilber FC, Singh SR, Zhao M and Hoffman RM: A combination of irinotecan/cisplatinum and irinotecan/temozolomide or tumor-targeting Salmonella typhimurium A1-R arrest doxorubicin- and temozolomide-resistant myxofibrosarcoma in a PDOX mouse model. Biochem Biophys Res Commun 505: 733739, 2018. PMID: 30292411. DOI: 10.1016/j.bbrc.2018.09.106

15 Igarashi K, Kawaguchi K, Kiyuna T, Murakami T, Miwa S, Nelson SD, Dry SM, Li Y, Singh AS, Kimura H, Hayashi K, Yamamoto N, Tsuchiya H, Eilber FC and Hoffman RM:
Temozolomide combined with irinotecan caused regression in an adult pleomorphic rhabdomyosarcoma patient-derived orthotopic xenograft (PDOX) nude-mouse model. Oncotarget 8: 7587475880, 2017. PMID: 29100276. DOI: 10.18632 /oncotarget.16548

16 Murakami T, Igarashi K, Kawaguchi K, Kiyuna T, Zhang Y, Zhao M, Hiroshima Y, Nelson SD, Dry SM, Li Y, Yanagawa J, Russell T, Federman N, Singh A, Elliott I, Matsuyama R, Chishima T, Tanaka K, Endo I, Eilber FC and Hoffman RM: Tumor-targeting Salmonella typhimurium A1-R regresses an osteosarcoma in a patient-derived xenograft model resistant to a molecular-targeting drug. Oncotarget 8: 8035-8042, 2017. PMID: 28030831. DOI: 10.18632/oncotarget.14040.

17 Igarashi K, Kiyuna T, Miyake K, Miyake M, Li Y, Nelson SD, Dry SM, Singh AS, Elliott IA, Russell TA, Eckardt MA, Yamamoto N, Hayashi K, Kimura H, Miwa S, Tsuchiya H, Eilber FC and Hoffman RM: Temozolomide combined with irinotecan regresses a cisplatinum-resistant relapsed osteosarcoma in a patient-derived orthotopic xenograft (PDOX) precision-oncology mouse model. Oncotarget 9: 7774-7781, 2018. PMID: 29487690. DOI: 10.18632/oncotarget. 22892

18 Igarashi K, Kawaguchi K, Murakami T, Kiyuna T, Miyake K, Nelson SD, Dry SM, Li Y, Yanagawa J, Russell TA, Singh AS, Yamamoto N, Hayashi K, Kimura H, Miwa S, Tsuchiya H, Eilber FC and Hoffman RM: Intra-arterial administration of tumor-targeting Salmonella typhimurium A1-R regresses a cisplatin-resistant relapsed osteosarcoma in a patient-derived orthotopic xenograft (PDOX) mouse model. Cell Cycle 16: 1164-1170, 2017. PMID: 28494180. DOI: 10.1080/15384101. 2017.1317417

19 Asano M, Matsui J, Towle MJ, Wu J, McGonigle S, DE Boisferon MH, Uenaka T, Nomoto K and Littlefield BA: Broadspectrum preclinical antitumor activity of eribulin (Halaven ${ }^{\circledR}$ ): combination with anticancer agents of differing mechanisms. Anticancer Res 38(6): 3375-3385, 2018. PMID: 29848686. DOI: 10.21873/anticanres.12604

20 Ito K, Hamamichi S, Abe T, Akagi T, Shirota H, Kawano S, Asano M, Asano O, Yokoi A, Matsui J, Umeda IO and Fujii H: Antitumor effects of eribulin depend on modulation of the tumor microenvironment by vascular remodeling in mouse models. Cancer Sci 108(11): 2273-2280, 2017. PMID: 28869796. DOI: $10.1111 /$ cas.13392
Received July 16, 2019

Revised July 28, 2019

Accepted July 30, 2019 